\title{
Mitigating Effect of Glycinebetaine Pretreatment on Drought Stress Responses of Creeping Bentgrass
}

\author{
Lu Gan \\ Institute of Turfgrass Science, Beijing Forestry University, Beijing 100083, \\ P.R. China
}

Xunzhong Zhang
Department of Crop and Soil Environmental Sciences, Virginia Tech,
Blacksburg, VA 24061

Silu Liu and Shuxia Yin ${ }^{1}$

Institute of Turfgrass Science, Beijing Forestry University, Beijing 100083, P.R. China

Additional index words. plant growth regulation, water stress, ameliorate, antioxidant enzyme, expression levels

\begin{abstract}
Turfgrass performance under drought stress is impeded by plant water deficit and oxidative damage, which might be improved by the external application of osmoprotectants. Creeping bentgrass (Agrostis stolonifera L.) is a valuable species for low-cut golf surfaces as a result of its high density and fine texture. However, weak tolerance to drought stress is a primary shortcoming. In this study, the effect of exogenous glycinebetaine (GB) pretreatment on mitigating the damage from drought stress in creeping bentgrass cultivar ' $T-1$ ' was evaluated. Pieces of creeping bentgrass sod were subjected to four treatments: 1) well-watered control, 2) well watered and sprayed with $100 \mathrm{~mm}$ GB, 3) drought stress, and 4) drought stress and sprayed with $100 \mathrm{~mm}$ GB. Drought stress resulted in a remarkable decrease in turf quality (TQ), relative water content (RWC), and chlorophyll content, with significant increases in superoxide anion content $\left(\mathrm{O}_{2}^{-}\right)$, malondialdehyde (MDA) content, superoxide dismutase (SOD), catalase (CAT), and peroxidase (POD) activity. In contrast, pretreatment with $100 \mathrm{~mm}$ GB decreased the $\mathrm{O}_{2}^{-}$ and MDA content in water-stressed plants, and increased turf quality, chlorophyll content, SOD, CAT, and POD activity. Meanwhile, the expression level of the $p s b A, S A M S 4, C M O$, and $A C S 1$ genes in leaf samples collected during the drought-stress stage was elevated in GB pretreatment. Notably, SAMS4 gene expression in GB pretreatment was significantly greater than in the untreated GB groups subjected to water stress. These results suggested that GB could mitigate the adverse effect of water stress on creeping bentgrass. The amelioration related strongly to the maintenance of the antioxidant enzyme system, accumulated endogenous compatible metabolites, and the elevation of gene expression levels. These findings lead us to conclude that GB pretreatment could be used as an ameliorative agent for creeping bentgrass against the deleterious effects of water stress.
\end{abstract}

Creeping bentgrass (Agrostis stolonifera L.) is one of the most important turfgrass species for putting greens because of its excellent tolerance to low mowing height (commonly, 5-8 mm) (Fagerness and Yelverton, 2001; Turgeon, 1991). As an important turfgrass for sports fields and lawns, it usually requires intensive management and a large amount of water (Warnke, 2003). Thus, drought stress seriously affects TQ and the service life of the lawn. Moreover, frequent

\footnotetext{
Received for publication 26 July 2018. Accepted for publication 12 Sept. 2018.

This study was supported by the National Natural Science Foundation of China (no. 31302016) and the Science and Technology Program of Shenzhen (JCYJ20160331151245672 and JSGG20160229155434792).

${ }^{1}$ Corresponding author. E-mail: yinsx369@bjfu. edu.cn.
}

low mowing causes a series of problems in turfgrass (poorly developed root system), which make the turf more vulnerable to drought stress. Drought stress, a major limiting factor for turfgrass management in many regions (Huang et al., 2014), results in a buildup of reactive oxidative species in plant cells and damages protein structures and cell membranes; drought-induced osmotic stress may also occur in most plants (Ashraf, 2010; Reddy et al., 2004). To cope with drought stress, plants store multiple groups of compatible metabolites such as betaines, proline, and sugar alcohols (Dawood et al., 2014; Hussain Wani et al., 2013). It has been well established that GB plays an important role in defensive mechanisms, maintaining osmotic balance; stabilizing the structure and function of proteins, enzymes, and membranes; and inducing resistance-related gene expression (Bohnert and Jensen, 1996; Hussain Wani et al., 2013; Rathinasabapathi, 2000; Saneoka et al.,
1995). It has been reported that exogenous application of GB could alleviate the damage of abiotic stress (i.e., salt, drought, and heat stress) in various crop plants and turfgrass (Burgess and Huang, 2014; Malekzadeh, 2015; Maziah and Teh, 2016; Raza et al., 2014). In addition, GB is also known to have a positive effect on perennial ryegrass under cadmium stress (Lou et al., 2015).

As one of the compatible solutes in plants, GB could protect membranes against oxidative damage by stimulating antioxidative enzymes such as SOD (EC 1.15.1.1), CAT (EC 1.11.1.6), and APX (EC 1.11.1.11) (Ma et al., 2004). In addition, both endogenous and exogenous GB could protect the structure and function of photosystem II (PSII), which is the most vulnerable to environmental stress, thus maintaining photosynthetic carbon assimilation to alleviate the decrease of photosynthetic capacity under stress (Ma et al., 2006; Wang et al., 2014). The expression level of the $p s b A$ gene, encoding the D1 protein, plays a key role in the structure of the PSII complex and is related to drought stress and GB application (Yamamoto et al., 2008). Research has shown that phytohormone biosynthetic and signaling pathways interact with metabolites and regulate the growth and development of plants under drought stress (Peleg and Blumwald, 2011). Some genes encode resistance-related enzymes in the biosynthesis of hormones. For example, the overexpression of 9-cis-epoxycarotenoid dioxygenase (NCED), a key enzyme in ABA biosynthesis, improved drought stress tolerance in transgenic arabidopsis plants (Iuchi et al., 2001). In addition, 1-aminocyclopropane carboxylic acid synthase is the first enzyme in the biosynthesis pathway of ethylene-the vital molecular substrate regulating photosynthesis and growth of plants under stressful environments (Larrainzar et al., 2014). Sadenosyl methionine synthase is the vital enzyme for the biosynthesis of GB and ethylene (Moffatt and Weretilnyk, 2001). So, for plants exposed to drought stress, exogenous GB has been suggested as a possible approach to protecting cellular structures and key metabolites (Ashraf and Foolad, 2007; Zhang et al., 2013).

With regard to previous studies of field crops, there is little information available in the literature on the alleviation effect of GB pretreatment on enzyme antioxidants, and the expression of related genes has not been elucidated in creeping bentgrass. With this aim in mind, the effects of exogenous GB on plant growth, osmolyte accumulation, the antioxidative defense system, and the molecular expression response to drought stress were investigated in creeping bentgrass. It is hypothesized that exogenous GB application may alleviate oxidative damage, with enhanced activities of enzyme antioxidants and gene expression levels. Moreover, the possible mechanism of the GB protective function on drought-stressed creeping bentgrass was examined.

\section{Materials and Methods}

Plant materials and growth conditions. Mature creeping bentgrass ' $\mathrm{T}-1$ ' sod in a 


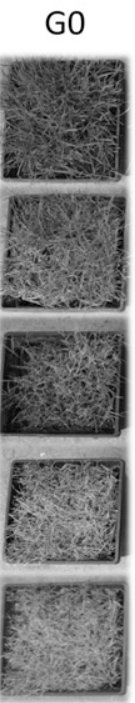

\section{G0}

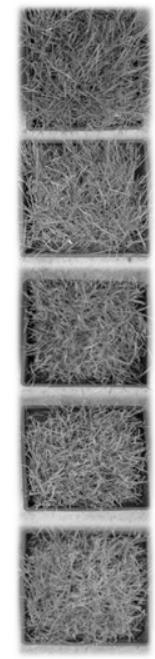

TO

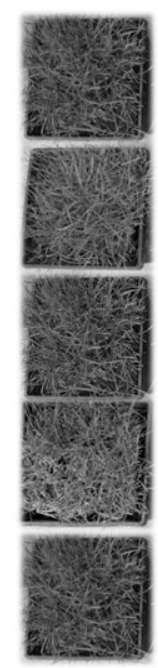

\section{G100}

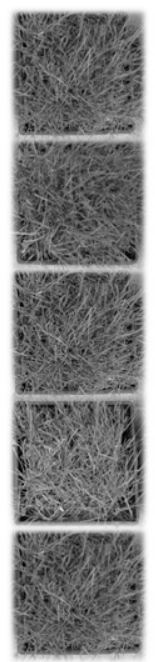

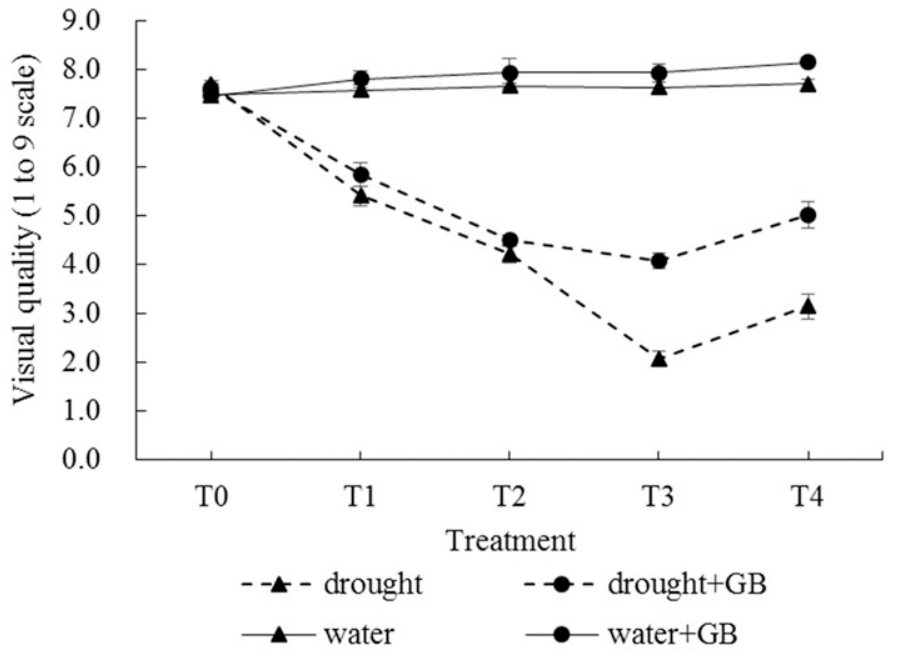

Fig. 1. Effects of glycinebetaine (GB) on turf quality of creeping bentgrass under drought stress and well-watered conditions. (A) Photos of plants during the water deficit treatment, from left to right, are the drought group, drought + GB group, water group, and water + GB group. (B) The value of visual turf quality. Each bar is the mean \pm SE of at least three replicates.

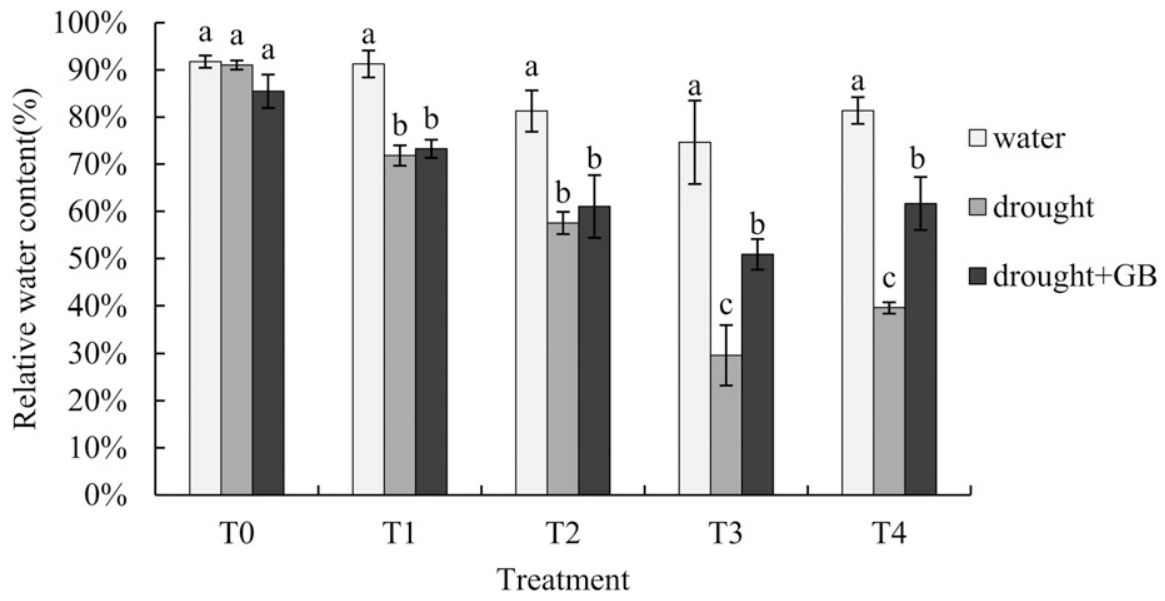

Fig. 2. Effects of glycinebetaine (GB) on leaf relative water content of creeping bentgrass in well-watered (control), drought, and drought + GB groups. Each bar is the mean \pm SE of at least three replicates. Bars with different letters are significantly different at $P<0.05$.

uniform size (length, $15 \mathrm{~cm}$; depth, $5 \mathrm{~cm}$ ) was collected from the field of the Turfgrass Research Station, Beijing Forestry University, Changping District, Beijing, China, and cultivated in plastic pots (height, $16.5 \mathrm{~cm}$; diameter, $16.5 \mathrm{~cm}$ ) containing sand and soil $(1: 2, \mathrm{v} / \mathrm{v})$. The plants grew in the growth chamber under the controlled conditions ( 300 $\mu \mathrm{mol} \cdot \mathrm{m}^{-2} \cdot \mathrm{s}^{-1}$ photosynthetic photon flux density light; $75 \%$ relative humidity; $25 / 20^{\circ} \mathrm{C}$ of continuous day/night temperatures, and a $12-/ 12-\mathrm{h}$ day/night cycle). A $50 \%$ Hoagland nutrient solution \% was applied weekly and the grass was mowed to a height of $5 \mathrm{~cm}$.

Treatments. To determine the optimum GB application rate for creeping bentgrass, a preliminary experiment was conducted with GB concentrations at 50,100, and $200 \mathrm{~mm}$. The grass treated with $100 \mathrm{~mm}$ GB showed the best performance under drought stress (data not shown). Yang et al. (2012) found that $100 \mathrm{~mm}$ was the best in perennial ryegrass. In the current study, before imposing a water deficit, an aqueous solution of $100 \mathrm{~mm}$ GB was sprayed on leaves until runoff was detected. The solution was sprayed at 5:00 PM for $3 \mathrm{~d}$ consecutively. Drought stress was induced by withholding watering after GB application. For wellwatered groups, pots were normally irrigated according to soil water content as determined by using an HH2 Moisture Meter (Delta-T Devices, Cambridge, UK); the soil water content was maintained at around 35\%. For water deficit treatments, irrigation was stopped after GB application to reduce soil water content gradually to $10 \%$. All plants were divided into four groups: 1) well watered without GB treatment (control or water group), 2) well watered with GB treatment (water + GB group; some index of this group is not shown in the following text because no significant difference was observed compared with the control group); 3) drought stressed without GB treatment (drought group), and 4) drought stress with GB treatment (drought + GB group). There were five replicate pots per treatment and the pots were assigned by random complete block design. For all treatments, leaf samples were collected at every level of drought stress, with the soil water content around $35 \%$, $22 \%, 14 \%, 10 \%$, and $32 \%$ ( $12 \mathrm{~h}$ after rewatering), representing no (T0), mild (T1), moderate (T2), and severe drought stress (T3), and then rewatered and maintained (T4), respectively (Supplemental Table 1). The fresh weight (FW) of the samples was determined immediately after collection; other leaf samples were stored at $-80{ }^{\circ} \mathrm{C}$. For gene expression analysis, the leaf samples were collected at T0, T1, T2, T3, and $\mathrm{T} 4$ (12 $\mathrm{h}$ after rewatering).

TQ - including turfgrass color, leaf texture, and uniformity - was rated visually on a scale of 1 to 9 (where 9 represents the best quality). All ratings were recorded at T0, T1, T2, T3, and T4 (4 d after rewatering) before collecting leaf samples for physiologic analysis

Determination of leaf $R W C$ and chlorophyll content. RWC was determined according to the formula: $\mathrm{RWC}=[(\mathrm{FW}-$ dry weight)/(turgid weight - dry weight)] $\times$ $100 \%$. Turgid weight was determined after leaves were immersed in water for $24 \mathrm{~h}$ at $25^{\circ} \mathrm{C}$. Dry weight was measured after ovendrying at $105{ }^{\circ} \mathrm{C}$ until a constant mass was reached (Hu et al., 2010).

Chlorophyll content was extracted by soaking $50 \mathrm{mg}$ of leaves in $8 \mathrm{~mL}$ acetone $(95 \%, v / v)$ that were then placed in the dark for $48 \mathrm{~h}$. Afterward, the homogenate was measured with a spectrophotometer at 665 , 649, and $470 \mathrm{~nm}$. Chlorophyll content was calculated using the formula of Arnon (1949).

Determination of $\mathrm{O}_{2}^{-}$production and MDA content. $\mathrm{O}_{2}^{-}$was measured as described by Li and Gong (2005), with some modifications. Frozen leaf segments of $0.1 \mathrm{~g}$ were 
ground and then homogenized with $1 \mathrm{~mL}$ of $65 \mathrm{~mm}$ potassium phosphate buffer $(\mathrm{pH} 7.8)$ and centrifuged at $5000 \mathrm{~g}$ for $10 \mathrm{~min}$. The incubation mixture contained $0.9 \mathrm{~mL}$ of $65 \mathrm{~mm}$ phosphate buffer $(\mathrm{pH} 7.8), 0.1 \mathrm{~mL}$ of $10 \mathrm{~mm}$ hydroxylamine hydrochloride, and $1 \mathrm{~mL}$ of the supernatant. After incubation at $25{ }^{\circ} \mathrm{C}$ for $20 \mathrm{~min}, 0.2 \mathrm{~mL}$ of $17 \mathrm{~mm}$ sulfanilamide and $7 \mathrm{~mm}$ alpha-naphthylamine were added to the incubation mixture, and reacted at $25{ }^{\circ} \mathrm{C}$ for $20 \mathrm{~min}$. Then, ethyl ether at the same volume was added and centrifuged at $1500 \mathrm{~g}$ for $5 \mathrm{~min}$. The absorbance in the aqueous solution was read at $530 \mathrm{~nm}$. A standard curve with $\mathrm{NO}_{2}^{-}$was used to calculate the product rate of $\mathrm{O}_{2}^{-}$from the chemical reaction of $\mathrm{O}_{2}^{-}$and hydroxylamine.

Oxidative damage to lipids was estimated by measuring the content of MDA in leaf segment homogenates. The MDA level was assayed using the $0.25 \%$ thiobarbituric acid reaction as described by Dhindsa et al. (1981). MDA content (measured in micromoles per gram $\mathrm{FW}$ ) was estimated according to the following formula: $\mathrm{MDA}=6.45 \times\left(\mathrm{A}_{532}-\right.$ $\left.\mathrm{A}_{600}\right)-0.56 \times \mathrm{A}_{450}$, where $\mathrm{A}_{532}, \mathrm{~A}_{600}$, and $\mathrm{A}_{450}$ represent the absorbance of the mixture at 532, 600, and $450 \mathrm{~nm}$, respectively.

Determination of betaine aldehyde dehydrogenase activity and endogenous $G B$ contents. Betaine aldehyde dehydrogenase activity (BADH) activity was determined using the method of Zhang et al. (2011), with some modifications. Frozen leaf samples ( $2 \mathrm{~g})$ were ground into homogenate with extraction solution containing $50 \mathrm{~mm}$ Tris- $\mathrm{HCl}, 1 \mathrm{~mm}$ ethylenediamine tetra-acetic acid (EDTA), and $5 \mathrm{~mm}$ DTT in liquid nitrogen, and then was centrifuged at $13,000 \mathrm{rpm}$ for $10 \mathrm{~min}$ at $4{ }^{\circ} \mathrm{C}$. The reaction system of BADH activity included $50 \mathrm{~mm}$ Tris- $\mathrm{HCl}, 1 \mathrm{~mm}$ EDTA, $5 \mathrm{~mm}$ DTT, $1 \mathrm{~mm}$ betaine aldehyde, $1 \mathrm{~mm}$ $\mathrm{NAD}$, and $1.0 \mathrm{mg}$ crude protein extract (calculated by the protein concentration of crude extraction according to the Bradford method). The mixed liquid was placed at $37{ }^{\circ} \mathrm{C}$ for $10 \mathrm{~min}$. BADH activity was detected at $340 \mathrm{~nm}$ by ultraviolet (UV) spectrophotometry.

The endogenous GB content was detected as described by Grieve and Grattan (1983), with some modification. Frozen samples $(0.5 \mathrm{~g})$ were ground with an extracting buffer of methanol:chloroform:water (ultrapure) $(12: 5: 3, \mathrm{v} / \mathrm{v} / \mathrm{v})$ and kept in warm distilled water $\left(70{ }^{\circ} \mathrm{C}\right)$ for $10 \mathrm{~min}$. The mixture was then centrifuged at $3000 \mathrm{~g}$ at $20{ }^{\circ} \mathrm{C}$ for $15 \mathrm{~min}$, and the upper aqueous solution was concentrated to dryness and dissolved in $30 \mathrm{~mL}$ of $1 \mathrm{M} \mathrm{HCl}$. The dilute hydrochloric acid solution was extracted twice by petroleum ether and decolored with activated charcoal. Crystals were dissolved in $70 \%$ acetone, and absorbance was read at 525 nm. Appropriate GB standards were used for calibration and calculation of GB concentration in unknown samples.

Assays of antioxidant enzyme activity. Frozen leaf samples of 0.5 to $0.8 \mathrm{~g}$ were excised and ground with a pestle in an icecold mortar with $8 \mathrm{~mL}$ extraction buffer

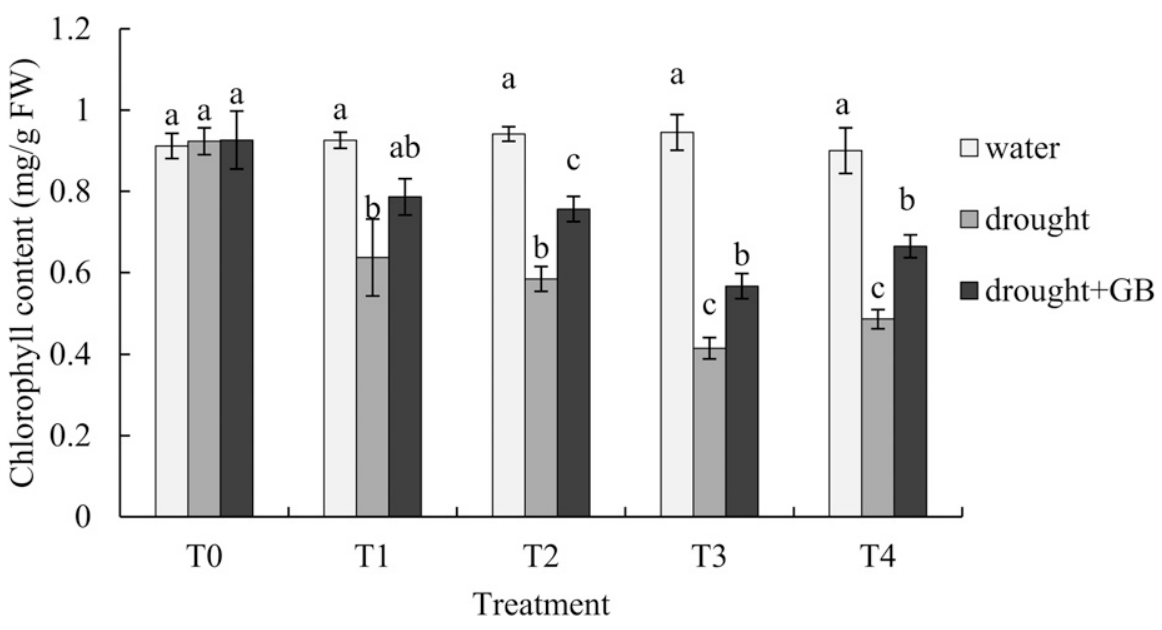

Fig. 3. Effects of glycinebetaine (GB) on leaf chlorophyll content of creeping bentgrass in well-watered (control), drought, and drought $+\mathrm{GB}$ groups. Each bar is the mean $\pm \mathrm{SE}$ of at least three replicates. Bars with different letters are significantly different at $P<0.05$. FW $=$ fresh weight.
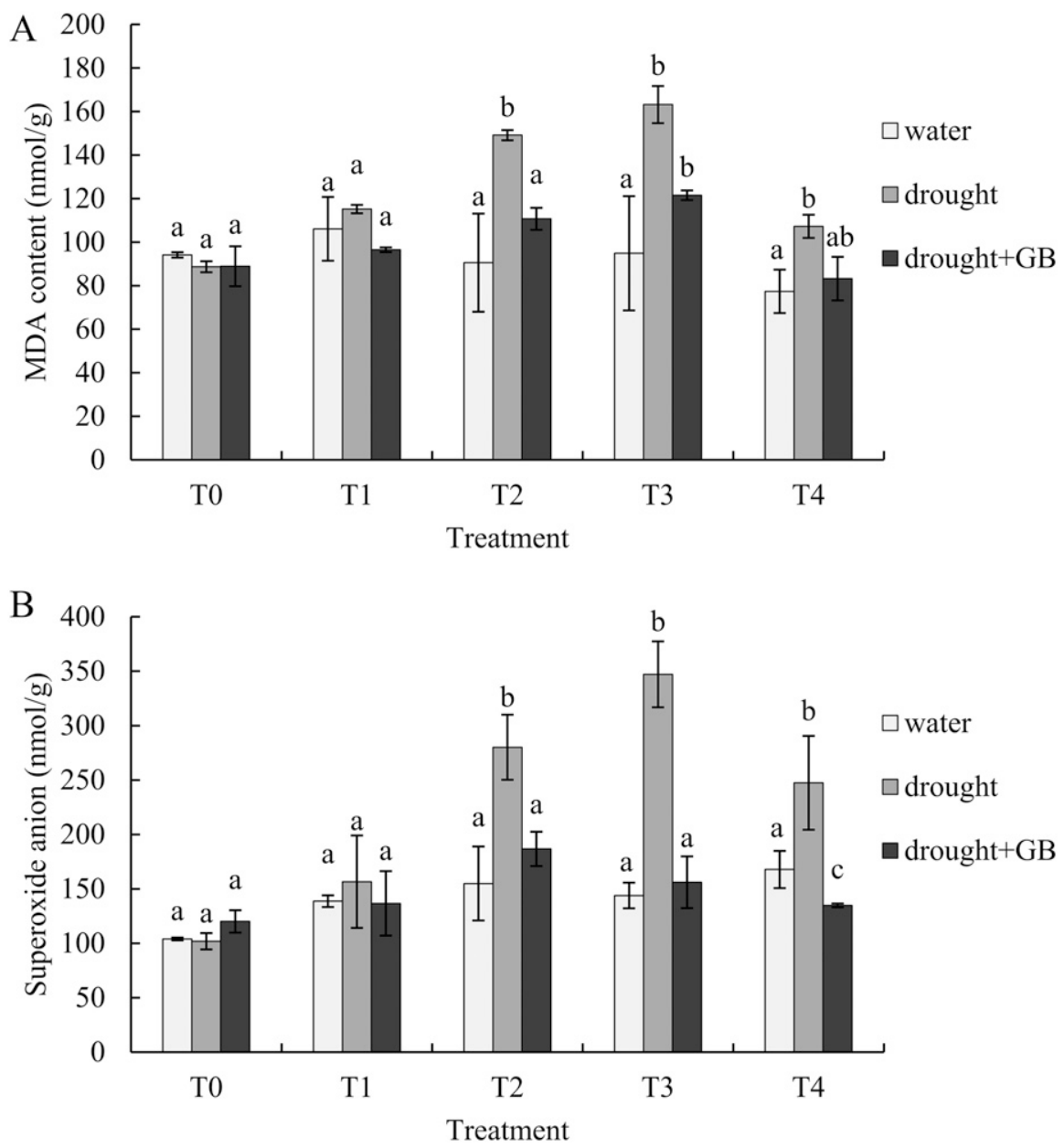

Fig. 4. Effects of glycinebetaine (GB) on leaf (A) malondialdehyde (MDA) and (B) superoxide anion content of creeping bentgrass in well-watered (control), drought, and drought + GB groups. Each bar is the mean \pm SE of at least three replicates. Bars with different letters are significantly different at $P<0.05$.

[50 mm potassium phosphate buffer ( $\mathrm{pH} 7.8)$, $0.1 \mathrm{~mm}$ EDTA, $0.5 \%(\mathrm{~m} / \mathrm{v})$ polyvinylpyrrolidone, and $0.1 \%$ Triton X-100]. The homogenates were filtered through four layers of gauze and then centrifuged at $12,000 \mathrm{~g}$ for $30 \mathrm{~min}$ at $4{ }^{\circ} \mathrm{C}$. The supernatants were used to assay antioxidative enzymatic activity.
SOD activity was determined as described by Zhang and Kirkham (1996). The activity of CAT and POD was detected by UV spectrophotometry and guaiacol (Chance and Maehly, 1955).

Determination of expression level of stress-related genes. Homologous sequences 
of stress-related genes (including $p s b A$, $C M O, S A M S 4$, and ACS1) in Hordeum vulgare, Brachypodium distachyon, and Setaria italica were downloaded from the National Center for Biotechnology Information (NCBI) nonreductant database. Based on these sequences, the conserved sequences of the previously mentioned stress-related genes (Supplemental Table 2) were amplified from creeping bentgrass using a homology cloning strategy with specified primers (Supplemental Table 3), and were then sent to a sequencing facility. Total RNA was extracted using the TRIzol reagent kit (Invitrogen), according to the manufacturer's recommendations for gene expression analysis. Total RNA quantity and purity were assessed using a NanoDrop 2000 spectrophotometer (Thermo). For complementary (cDNA) synthesis, a total of $0.5 \mu \mathrm{g}$ RNA was reverse-transcribed using an oligo (dT) primer and PrimeScript RT Reagent Kit (Perfect Real Time) (Takara, Japan). Real-time polymerase chain reaction (PCR) was performed using a Bio-Rad CFX Connect system and Bio-Rad CFX manager software (version 3.1) with the UltraSYBR mix (KangWei, China) according to the manufacturer's guidelines. The primer pairs for quantitative reverse transcription-polymerase chain reaction (qRT-PCR) are shown in Supplemental Table 4. Briefly, qRT-PCR was performed in a total volume of $20 \mu \mathrm{L}$ containing $2 \mu \mathrm{L}$ cDNA, $0.5 \mu \mathrm{L}$ of each primer, and $10 \mu \mathrm{L}$ of SYBR mix. The cycling program was as follows: $95^{\circ} \mathrm{C}$ for $5 \mathrm{~s}$, followed by 40 cycles at $95^{\circ} \mathrm{C}$ for $30 \mathrm{~s}$, annealing at $58^{\circ} \mathrm{C}$ for $30 \mathrm{~s}$, and extension at $72{ }^{\circ} \mathrm{C}$ for $1 \mathrm{~min}$. The relative expression of each gene was evaluated by the expression of the internal standard AsActin (geneID: 443298220) using the comparative CT method $\left(2^{-\Delta \Delta C T}\right)$. All quantification measurements were carried out three times.

Experimental design and statistical analysis. Each pot in one treatment was regarded as one replicate, and the experimental results were presented with the SE of five replicates. These data were subject to analysis of variance and Fisher's least significant difference test, if the $\mathrm{F}$ test was significant at $P=0.05$

\section{Results}

Turf quality. Drought stress decreased TQ (Fig. 1). Under the treatment of severe drought followed by rewatering, GB application alleviated TQ decline significantly (final TQ rating, 5.00) compared with the treatment of drought stress without GB (final TQ rating, 3.13; $P<0.05)$. But, GB pretreatment did not have a significant impact on TQ under mild and moderate drought stress.

Relative water content. As shown in Fig. 2, drought stress had a negative effect on RWC of leaves and reached its lowest value at the point of severe drought stress (T3). In creeping bentgrass, the GB-treated group exhibited a slower decrease in RWC during drought stress. From moderate to severe drought stress, for example, RWC
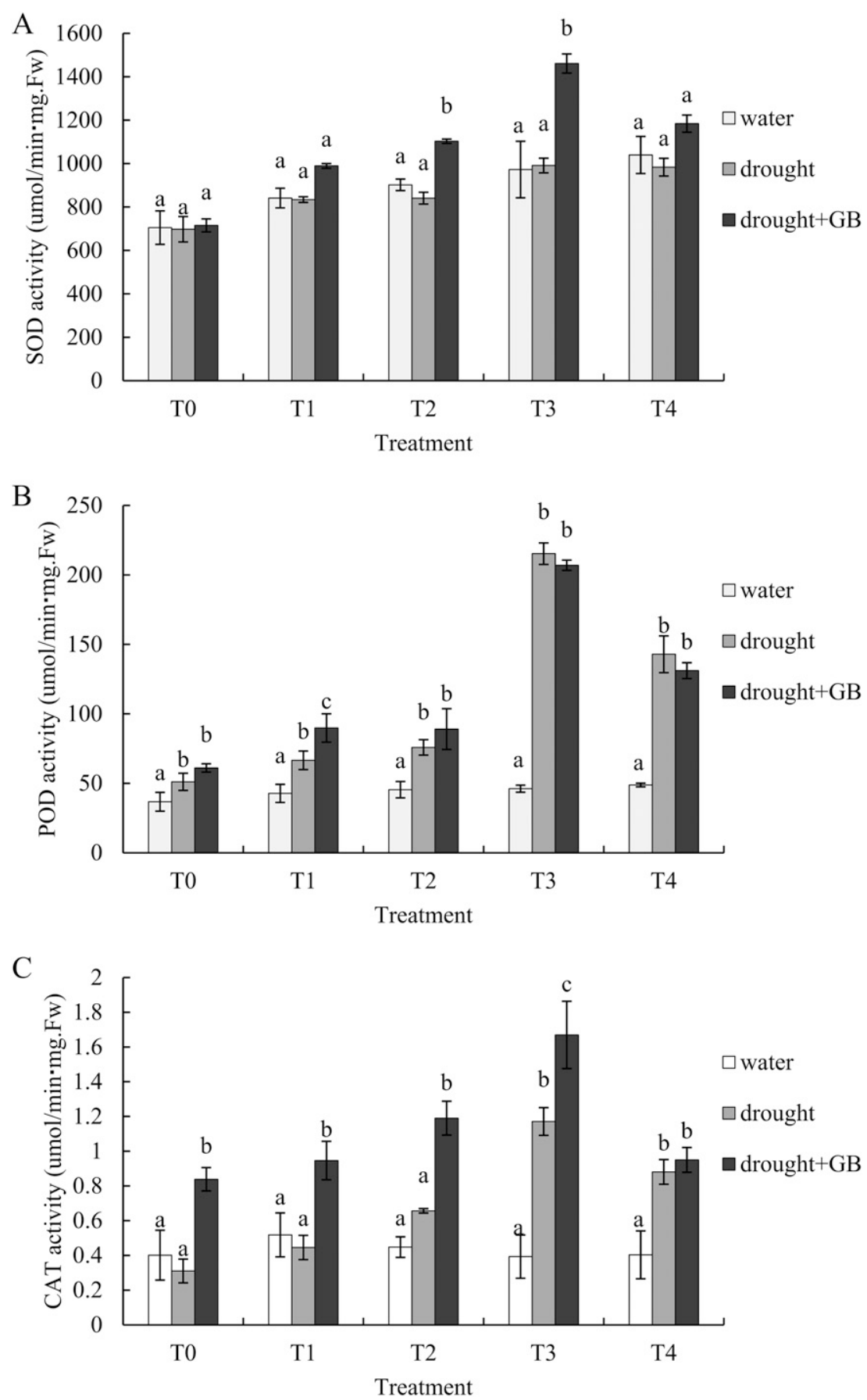

Fig. 5. Effects of glycine betaine (GB) on activities of (A) superoxide dismutase (SOD), (B) peroxidase (POD), and (C) catalase (CAT) of creeping bentgrass in well-watered (control), drought, and drought + $\mathrm{GB}$ groups. Each bar is the mean $\pm \mathrm{SE}$ of at least three replicates. Bars with different letters are significantly different at $P<0.05$.

decreased by $27.95 \%$ in the drought group, but only by $10.16 \%$ in the drought + GB group, which illustrates that GB pretreatment suppressed the downturn of plant RWC effectively. We concluded that exogenous GB could improve the osmotic regulation ability of leaf blade cells under drought stress and, consequently, enhanced water retention.

Chlorophyll content. Under drought stress, the chlorophyll content was also greater in the drought $+\mathrm{GB}$ pretreatment compared with the drought group without GB (Fig. 3). These results illustrate that GB pretreatment could alleviate the decline of chlorophyll content $(P<0.05)$. For instance, during the initial stages of drought stress (from T0 to T2), the chlorophyll content decreased by $36.74 \%$ in the drought group, but only by $18.34 \%$ in the drought + GB group. Moreover, it appeared that the chlorophyll content increment from severe drought stress to rewatering in the drought + GB group $\left(0.098 \mathrm{mg} \cdot \mathrm{g}^{-1} \mathrm{FW}\right)$ was greater than that in the drought group $\left(0.07 \mathrm{mg} \cdot \mathrm{g}^{-1}\right.$ FW), which demonstrates that GB pretreatment could improve the chlorophyll content 
accumulation to support photosynthesis under drought stress and rewatering. This means the application of exogenous GB could contribute to plant recovery from drought damage after rewatering.

$M A D$ and $\mathrm{O}_{2}$ content. Water stress affected membrane integrity as a result of increased lipid peroxidation determined as the content of MDA. Under drought stress, MDA content was significantly greater than that in the well-watered group ( $P<0.05$; Fig. 4A). Foliar application of exogenous GB reduced MDA content (lipid peroxidation) in creeping bentgrass exposed to water stress compared with the drought-withoutGB treatment. The greatest reduction $(25.77 \%$, $P<0.05)$ was observed for the moderate stage drought (T2). Furthermore, MDA content decreased dramatically after rewatering. These results demonstrate that GB pretreatment could reduce cell membrane damage under drought stress and contribute to repairing the damage resulting from drought stress.

Compared with well-watered groups, $\mathrm{O}_{2-}$ content under drought stress increased significantly, especially in drought-without-GB treatment (Fig. 4B). However, $\mathrm{O}_{2-}$ content in the GB pretreatment group declined gradually after the peak of moderate drought stress-from 186.76 to $156.10 \mathrm{nmol} \cdot \mathrm{g}^{-1}$, which indicates that GB pretreatment reduced the production of $\mathrm{O}_{2-}$.

Antioxidant enzyme activities. SOD activity in the GB pretreatment group increased gradually with drought stress extension, although drought stress did not accelerate the accumulation of SOD (Fig. 5A). The increased amplitude in SOD activity in drought + GB treatment increased by $47.42 \%$ compared with the untreated group under severe drought, which indicates that exogenous GB pretreatment could improve SOD activity of creeping bentgrass under drought stress.

POD activity under drought stress increased significantly compared with the well-watered group, but it was remarkable that no significant difference was observed between GB-treated and GB-untreated plants during drought stress, except at the point of mild drought stress (Fig. 5B).

As shown in Fig. 5C, drought stress resulted in a significant accumulation in CAT at the point of severe drought stress and rewatering. With the drought stress extension, CAT activity increased accordingly. Compared with untreated plants during drought stress, CAT activity in GB pretreated group increased by $111.11 \%, 80.30 \%$, and $42.74 \%$, respectively, at the stages of mild (T1), moderate (T2), and severe drought (T3). This result shows that it was one of the most important ways that CAT activity increased during the early stage of drought in order that the plants adapt to the stress.

$B A D H$ activity and endogenous $G B$ contents. Under drought stress, both endogenous GB content and BADH activity increased with drought extension. Furthermore, both were significantly greater in the GB pretreatment group than in the untreated group $(P<0.05)$ during the entire period of drought stress (Fig. 6). After rewatering, both BADH activity
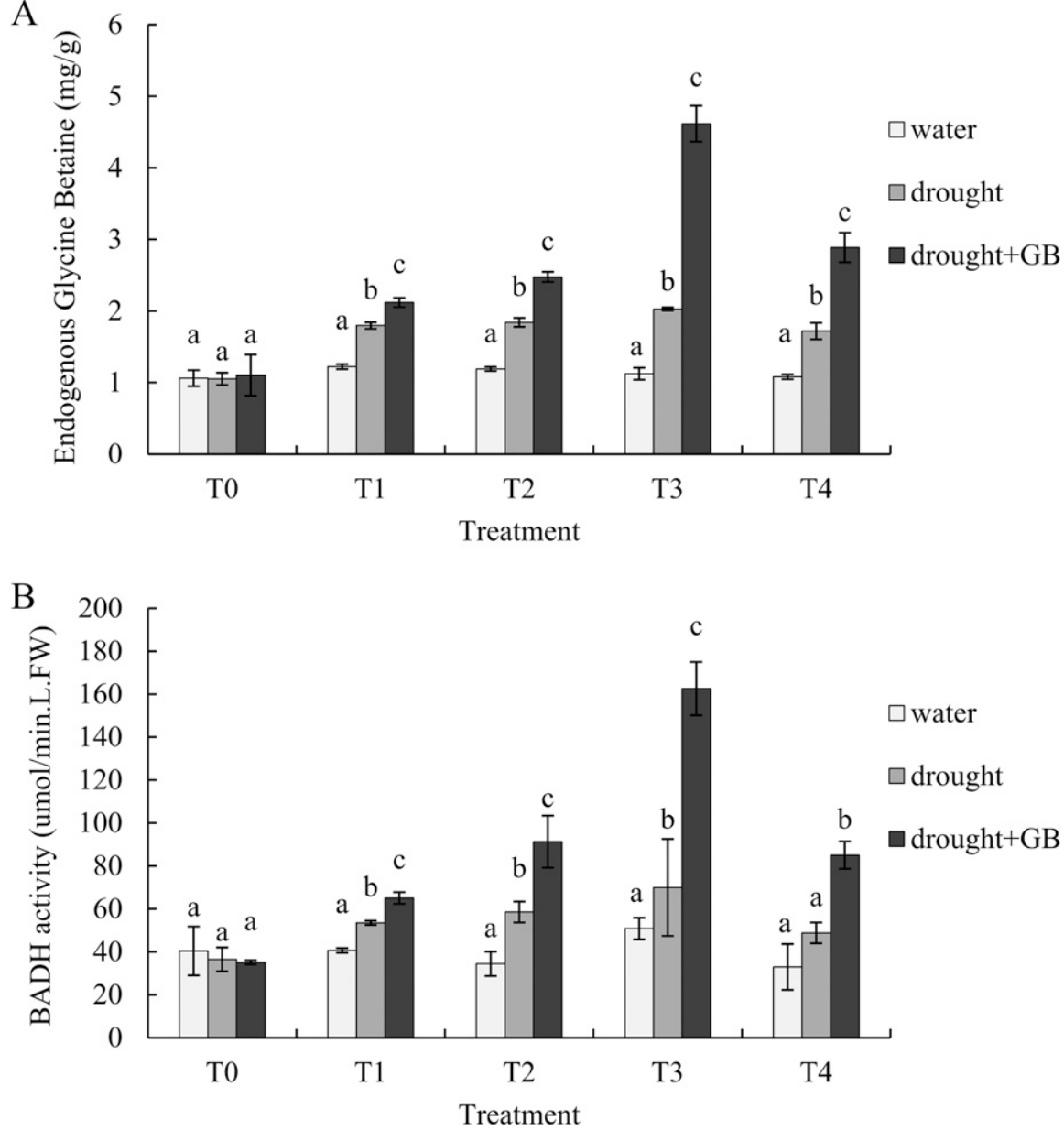

Fig. 6. Effects of glycine betaine (GB) on (A) GB level and (B) betaine aldehyde dehydrogenase (BADH) activity of creeping bentgrass in well-watered (control), drought, and drought + GB groups. Each bar is the mean \pm SE of at least three replicates. Bars with different letters are significantly different at $P<$ 0.05 .

and endogenous GB content declined, but was still greater than in the well-watered group. Basically, under drought stress, the trend of influence of exogenous GB on BADH activity and GB level of creeping bentgrass was almost in line. These results indicate that GB pretreatment increased endogenous GB content by elevating BADH activity, and then the osmotic potential of plant leaves increased as a consequence.

$q R T-P C R$ of stress-related gene transcripts. Under drought stress, the expression level of the $p s b A$ gene decreased with extended drought, but GB pretreatment improved gene expression (Fig. 7A). Notably, the expression levels were significantly greater than the untreated plants $(P<0.05)$ during mild (T1) and moderate (T2) drought. With regard to $S A M S 4$, transcriptional abundance accumulated in the GB pretreatment groups at T0 (no drought stress), then increased significantly more than in the untreated group during the whole period of drought stress (Fig. 7B). Similarly, the transcription level of the $A C S 1$ and $C M O$ genes in the drought + GB treatment was also greater than in untreated group and maintained a high level after rewatering (Fig. 7C and D). Different enhanced expression levels among
$S A M S 4, A C S 1$, and $C M O$ in the GB pretreated group indicated that $S A M S 4$ plays an essential role in the biosynthesis of endogenous GB and ethylene.

\section{Discussion}

Drought is one of the main environmental stresses that affect plant growth and development. The development of plants tolerant to environment stresses and searching for suitable stress alleviators are promising avenues of research. Many investigators have identified the positive effects of GB in plant growth under conditions of stress, including cellular osmotic adjustment, protection of membrane integrity, and scavenging of reactive oxygen species. The endogenous GB level in most plants under natural conditions is far less than the level that affects plant growth and development in natural accumulation as a result of the lack of available choline or because of reduced transport of choline to chloroplasts (Ma et al., 2006; Wang et al., 2014). However, GB is able to accumulate in response to stress conditions. In the current study, drought stress provoked the accumulation of endogenous GB in creeping bentgrass, which correlated with stress intensity (Fig. 6A). 

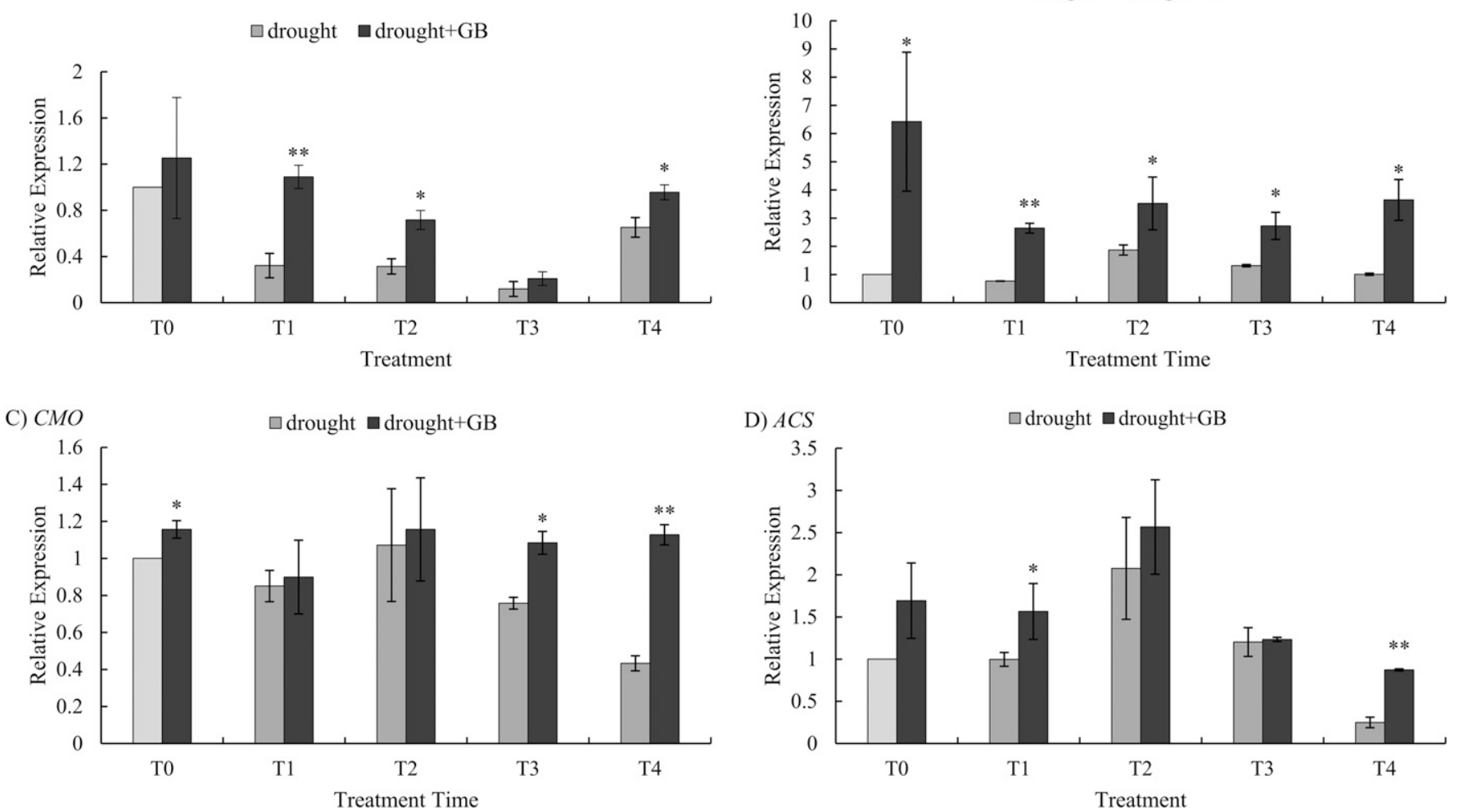

Fig. 7. Effects of glycine betaine (GB) on expression level of stress-related gene transcript of creeping bentgrass under drought stress with GB pretreatment (drought + GB group) and GB nontreatment (drought group). (A) Relative expression of the psbA gene. (B) Relative expression of the SAMS4 gene. (C) Relative expression of the $C M O$ gene. (D) Relative expression of the $A C S 1$ gene. The GB-untreated group at $0 \mathrm{~d}$ (no drought stress) is the control. An asterisk represents significant differences, with the corresponding control value at $* P<0.05$ and $* * P \leq 0.01$.

Lower accumulation of endogenous GB did not contribute to improved stress tolerance. However, the foliar application of exogenous GB helped reduce the adverse effects of drought stress. In the current study, RWC and TQ decreased rapidly under drought stress, which indicates that water balance and physiologic functioning were broken in mesophyll cells during drought stress. However, GB pretreated plants exhibited a slower decrease in RWC and $\mathrm{TQ}$, and developed wilting symptoms much later than untreated plants. In addition, drought + GB plants showed a better ability to recover from wilting after the removal of drought stress. It was illustrated that GB accumulation enhanced osmoregulation and relieved damage to leaf cells. Furthermore, drought stress induced a large number of reactive oxygen and free radicals, which resulted in the destruction of the active oxygen removal system and membrane lipid peroxidation (Jia et al., 2013; Zhou et al., 2013). The current study also demonstrates that drought stress resulted in a surge in $\mathrm{O}_{2}{ }^{-}$ and MDA content, both of which are important indicators of the degree of damage to cell membranes. Exogenous GB slowed the increase of $\mathrm{O}_{2}^{-}$and MDA. In addition, the activity of SOD, POD, and CAT, which eliminated excessive oxidation products and prevented free radical production, were increased under drought stress and further enhanced by GB pretreatment. These results demonstrate that GB pretreatment can im- prove the activity of antioxidant enzymes, help prevent reactive oxygen species and oxidative damage, alleviate membrane lipid peroxidation, and thus maintain the integrity and function of cell membranes (DaCosta and Huang, 2007; Hussain Wani et al., 2013). It has been reported that these mitigations of GB pretreatment attributed to the wheat seedlings in the oxidation reaction under drought stress (He et al., 2011).

GB is thought to play an adaptive role in stressed plants, but it did not accumulate in sufficient amounts to help avert the adverse effects of drought stress. Therefore, different approaches have been used to increase GB content in plants under stress conditions to increase their stress tolerance. In the current study, GB pretreated creeping bentgrass stimulated an accumulation of GB (Fig. 6A), and the application of exogenous GB in general enhanced plant growth and tolerance. In addition to its protective roles in enzyme and membrane integrity, the expression of related genes is also affected by exogenous GB, and these genes are mainly involved in the biosynthesis and metabolism pathway of GB and phytohormones. It has been shown that $C M O$ plays an important role in the pathway of glycine betaine synthesis (Burnet et al., 1995) and responses to abiotic stresses in rice (Luo et al., 2012), which are consistent with our results. Our study showed that the upregulated expression of the $C M O$ gene in drought-stressed plants with GB pretreatment was concomitant with the accumulation of exogenous glycine betaine and an increase in BADH activity. In addition, Sadenosyl methionine (SAM) is an essential precursor in successive steps to produce choline by transmethylation reactions (Moffatt and Weretilnyk, 2001), and ethylene by ACC synthase (Wang et al., 2002). Apelbaum and Yang (1981) indicated that drought stress stimulated ethylene production in wheat, but it did not affect the level of SAM, and it was assumed that the step converting SAM to ACC was rate limiting, whereas the step converting methionine to SAM was not rate limiting. In our study, the expression level of SAMS4 catalyzing methionine to SAM in GB-treated plants was significantly greater than in the untreated groups under drought stress, whereas $A C S 1$ and $C M O$ gene expression was slightly greater than in the untreated group at T0 (no drought stress). Hence, these data are consistent with the view that maintaining a stable level of SAM by enhancing its rate of synthesis must be essential when physiologic conditions demand it, as in the case of GB under drought stress. In addition, the drought resulted in a photosynthetic decline and osmotic stress, of which the D1 protein encoded by the chloroplast $p s b A$ gene was targeted in the damage course of PSII (Zhao et al., 2010). Excessive accumulation of GB enhances photosynthesis tolerance to drought in wheat flag leaves (Wang et al., 2014), and exogenous GB treatment was seen to alleviate suppressed psbA expression under drought stress (Hou et al., 2013). Attention should also be paid to the fact that, in our study, the chlorophyll content and $p s b A$ gene expression were 
decreased under drought stress, whereas GB pretreatment mitigated this effect.

In summary, drought stress severely hampers creeping bentgrass growth and TQ, but pretreatment with GB effectively ameliorates the adverse effects of drought stress. Foliar application of $100 \mathrm{~mm}$ GB could remove excessive reactive oxygen; retard the decrease of RWC and chlorophyll content by regulating antioxidant enzyme activities under drought stress; enhance the transcriptional level of psbA, SAMS4, CMO, and ACS1 to improve drought tolerance of creeping bentgrass. Because creeping bentgrass is the primary species used for golf greens and other high-input turf areas, it is necessary to maintain its high quality under various stresses. Before the onset of drought stress, exogenous GB applied (probably in a role of fertilizer) onto the grasses could improve the drought tolerance of creeping bentgrass, thus maintaining the high quality of turf during drought stress.

\section{Literature Cited}

Apelbaum, A. and S.F. Yang. 1981. Biosynthesis of stress ethylene induced by water deficit. Plant Physiol. 68:594-596.

Arnon, D.I. 1949. Copper enzymes in isolated chloroplasts: Polyphenoloxidase in Beta vulgaris. Plant Physiol. 24:1.

Ashraf, M. 2010. Inducing drought tolerance in plants: Recent advances. Biotechnol. Adv. 28:169-183.

Ashraf, M. and M. Foolad. 2007. Roles of glycine betaine and proline in improving plant abiotic stress resistance. Environ. Expt. Bot. 59:206-216.

Bohnert, H.J. and R.G. Jensen. 1996. Strategies for engineering water-stress tolerance in plants. Trends Biotechnol. 14:89-97.

Burgess, P. and B. Huang. 2014. Effects of sequential application of plant growth regulators and osmoregulants on drought tolerance of creeping bentgrass (Agrostis stolonifera). Crop Sci. 54:837-844.

Burnet, M., P.J. Lafontaine, and A.D. Hanson. 1995. Assay, purification, and partial characterization of choline monooxygenase from spinach. Plant Physiol. 108:581-588.

Chance, B. and A. Maehly. 1955. Assay of catalases and peroxidases, p. 764-775. Methods in Enzymology. Academic Press.

Dacosta, M. and B. Huang. 2007. Changes in antioxidant enzyme activities and lipid peroxidation for bentgrass species in response to drought stress. J. Amer. Soc. Hort. Sci. 132:319-326.

Dawood, M., H. Taie, R. Nassar, M. Abdelhamid, and U. Schmidhalter. 2014. The changes induced in the physiological, biochemical and anatomical characteristics of Vicia faba by the exogenous application of proline under seawater stress. S. Afr. J. Bot. 93:54-63.

Dhindsa, R.S., P. Plumb-Dhindsa, and T.A. Thorpe. 1981. Leaf senescence: Correlated with increased levels of membrane permeability and lipid peroxidation, and decreased levels of superoxide dismutase and catalase. J. Expt. Bot. 32:93-101.

Fagerness, M.J. and F.H. Yelverton. 2001. Plant growth regulator and mowing height effects on seasonal root growth of penncross creeping bentgrass. Crop Sci. 41:1901-1905.

Grieve, C. and S. Grattan. 1983. Rapid assay for determination of water soluble quaternary ammonium compounds. Plant Soil 70:303-307.
He, C., W. Zhang, Q. Gao, A. Yang, X. Hu, and J. Zhang. 2011. Enhancement of drought resistance and biomass by increasing the amount of glycine betaine in wheat seedlings. Euphytica 177:151-167.

Hou, P., J. Ma, P. Zhao, H. Zhang, H. Zhao, H. Liu, Y. Zhao, Y. Wang, J. Ma, and P. Zhao. 2013. Effects of betaine on chloroplast protective enzymes and $p s b A$ gene expression in wheat seedlings under drought stress. Acta Agronomica Sinica 39:1319-1324.

Hu, L., Z. Wang, and B. Huang. 2010. Diffusion limitations and metabolic factors associated with inhibition and recovery of photosynthesis from drought stress in a $\mathrm{C} 3$ perennial grass species. Physiol. Plant. 139:93-106.

Huang, B., M. Dacosta, and Y. Jiang. 2014. Research advances in mechanisms of turfgrass tolerance to abiotic stresses: From physiology to molecular biology. Crit. Rev. Plant Sci. 33:141-189.

Hussain Wani, S., N. Brajendra Singh, A. Haribhushan, and J. Iqbal Mir. 2013. Compatible solute engineering in plants for abiotic stress tolerance: Role of glycine betaine. Curr. Genomics 14:157-165.

Iuchi, S., M. Kobayashi, T. Taji, M. Naramoto, M. Seki, T. Kato, S. Tabata, Y. Kakubari, K. Yamaguchi-Shinozaki, and K. Shinozaki. 2001. Regulation of drought tolerance by gene manipulation of 9-cis-epoxycarotenoid dioxygenase, a key enzyme in abscisic acid biosynthesis in Arabidopsis. Plant J. 27:325-333.

Jia, X.-J., L.-H. Dong, C.-B. Ding, X. Li, and M. Yuan. 2013. Effects of drought stress on reactive oxygen species and their scavenging systems in Chlorophytum capense var. medio-pictum leaf. Acta Prataculturae Sinica 22:248-255.

Larrainzar, E., J.A. Molenaar, S. Wienkoop, E. GilQuintana, B. Alibert, A.M. Limami, C. ArreseIgor, and E.M. González. 2014. Drought stress provokes the down-regulation of methionine and ethylene biosynthesis pathways in $M$. edicago truncatula roots and nodules. Plant Cell Environ. 37:2051-2063.

Li, Z.-G. and M. Gong. 2005. Improvement of measurement method of polyphenol oxidase activities in plant. J. Yunnan Normal Univ 1:44 49 .

Lou, Y., Y. Yang, L. Hu, H. Liu, and Q. Xu. 2015. Exogenous glycinebetaine alleviates the detrimental effect of $\mathrm{Cd}$ stress on perennial ryegrass. Ecotoxicology 24:1330-1340.

Luo, D., X. Niu, J. Yu, J. Yan, X. Gou, B.-R. Lu, and Y. Liu. 2012. Rice choline monooxygenase (OsCMO) protein functions in enhancing glycine betaine biosynthesis in transgenic tobacco but does not accumulate in rice (Oryza sativa $\mathrm{L}$. ssp. japonica). Plant Cell Rpt. 31:1625-1635.

Ma, Q.-Q., W. Wang, Y.-H. Li, D.-Q. Li, and Q. Zou. 2006. Alleviation of photoinhibition in drought-stressed wheat (Triticum aestivum) by foliar-applied glycinebetaine. J. Plant Physiol. 163:165-175.

Ma, Q., Q. Zou, Y. Li, D. Li, and W. Wang. 2004. Amelioration of the water status and improvement of the anti-oxidant enzyme activities by exogenous glycinebetaine in water-stressed wheat seedlings. Zuo Wu Xue Bao 30:321-328.

Malekzadeh, P. 2015. Influence of exogenous application of glycinebetaine on antioxidative system and growth of salt-stressed soybean seedlings (Glycine max L.). Physiol. Mol. Biol. Plants 21:225-232.

Maziah, M. and C. Teh. 2016. Exogenous application of glycine betaine alleviates salt induced damages more efficiently than ascorbic acid in in vitro rice shoots. Austral. J. Basic Appl. Sci. 10:58-65.
Moffatt, B.A. and E.A. Weretilnyk. 2001. Sustaining S-adenosyl-1-methionine-dependent methyltransferase activity in plant cells. Physiol. Plant. 113:435-442.

Peleg, Z. and E. Blumwald. 2011. Hormone balance and abiotic stress tolerance in crop plants. Curr. Opin. Plant Biol. 14:290-295.

Rathinasabapathi, B. 2000. Metabolic engineering for stress tolerance: Installing osmoprotectant synthesis pathways. Ann. Bot. (Lond.) 86:709-716.

Raza, M., M. Saleem, G. Shah, I. Khan, and A. Raza. 2014. Exogenous application of glycinebetaine and potassium for improving water relations and grain yield of wheat under drought. J. Soil Sci. Plant Nutr. 14:348-364.

Reddy, A.R., K.V. Chaitanya, and M. Vivekanandan. 2004. Drought-induced responses of photosynthesis and antioxidant metabolism in higher plants. J. Plant Physiol. 161:1189-1202.

Saneoka, H., C. Nagasaka, D.T. Hahn, W.-J. Yang, G.S. Premachandra, R.J. Joly, and D. Rhodes. 1995. Salt tolerance of glycinebetaine-deficient and -containing maize lines. Plant Physiol. 107:631-638.

Turgeon, A.J. 1991. Turfgrass management. Prentice-Hall, Inc., Upper Saddle River, NJ.

Wang, K.L.-C., H. Li, and J.R. Ecker. 2002. Ethylene biosynthesis and signaling networks. Plant Cell 14:S131-S151.

Wang, G.-P., F.-X. Tian, M. Zhang, and W. Wang. 2014. The overaccumulation of glycinebetaine alleviated damages to PSII of wheat flag leaves under drought and high temperature stress combination. Acta Physiol. Plant. 36:2743-2753.

Warnke, S. 2003. Creeping bentgrass (Agrostis stolonifera L.), p. 175-185. Turfgrass biology, genetics, and breeding. Wiley, Hoboken, NJ.

Yamamoto, Y., R. Aminaka, M. Yoshioka, M. Khatoon, K. Komayama, D. Takenaka, A. Yamashita, N. Nijo, K. Inagawa, and N. Morita. 2008. Quality control of photosystem II: Impact of light and heat stresses. Photosynth. Res. 98:589-608

Yang, Z., J. Yu, E. Merewitz, and B. Huang. 2012. Differential effects of abscisic acid and glycine betaine on physiological responses to drought and salinity stress for two perennial grass species. J. Amer. Soc. Hort. Sci. 137:96106.

Zhang, L., M. Gao, L. Zhang, B. Li, M. Han, A.K. Alva, and M. Ashraf. 2013. Role of exogenous glycinebetaine and humic acid in mitigating drought stress-induced adverse effects in Malus robusta seedlings. Turk. J. Bot. 37:920-929.

Zhang, J. and M. Kirkham. 1996. Enzymatic responses of the ascorbate-glutathione cycle to drought in sorghum and sunflower plants. Plant Sci. 113:139-147.

Zhang, N., H.-J. Si, G. Wen, H.-H. Du, B.-L. Liu, and D. Wang. 2011. Enhanced drought and salinity tolerance in transgenic potato plants with a BADH gene from spinach. Plant Biotechnol. Rpt. 5:71-77.

Zhao, P., L. Wang, H. Zhao, S. Liang, S. Lv, X. Qu, and Y. Wang. 2010. Regulation of exogenous salicylic acid on expression of chloroplast gene $p s b A$ in wheat leaves under heat and high irradiance. Plant Physiol. Commun.. 46:537-540.

Zhou, L., P. Yan, and S. Xing-Yuan. 2013. Physiological responses of white clover by different leaf types associated with anti-oxidative enzyme protection and osmotic adjustment under drought stress. Acta Prataculturae Sinica 22 (02):257-263. 